\title{
The role of hot electron refluxing in laser-generated $K$-alpha sources
}

P. Neumayer, B. Aurand, M. Basko, B. Ecker, P. Gibbon, D. C. Hochhaus, A. Karmakar, E. Kazakov, T. Kühl, C. Labaune, O. Rosmej, An. Tauschwitz, B. Zielbauer, and D. Zimmer

Citation: Physics of Plasmas 17, 103103 (2010); doi: 10.1063/1.3486520

View online: https://doi.org/10.1063/1.3486520

View Table of Contents: http://aip.scitation.org/toc/php/17/10

Published by the American Institute of Physics

\section{Articles you may be interested in}

High-intensity laser interactions with mass-limited solid targets and implications for fast-ignition experiments on OMEGA EP

Physics of Plasmas 14, 056301 (2007); 10.1063/1.2472371

A study of picosecond laser-solid interactions up to $10^{19} \mathrm{~W} \mathrm{~cm}^{-2}$

Physics of Plasmas 4, 447 (1997); 10.1063/1.872103

Hot surface ionic line emission and cold K-inner shell emission from petawatt-laser-irradiated Cu foil targets Physics of Plasmas 13, 043102 (2006); 10.1063/1.2188912

High-energy $K \alpha$ radiography using high-intensity, short-pulse lasers

Physics of Plasmas 13, 056309 (2006); 10.1063/1.2178775

High energy proton acceleration in interaction of short laser pulse with dense plasma target

Physics of Plasmas 10, 2009 (2003); 10.1063/1.1556298

Energetic proton generation in ultra-intense laser-solid interactions

Physics of Plasmas 8, 542 (2001); 10.1063/1.1333697

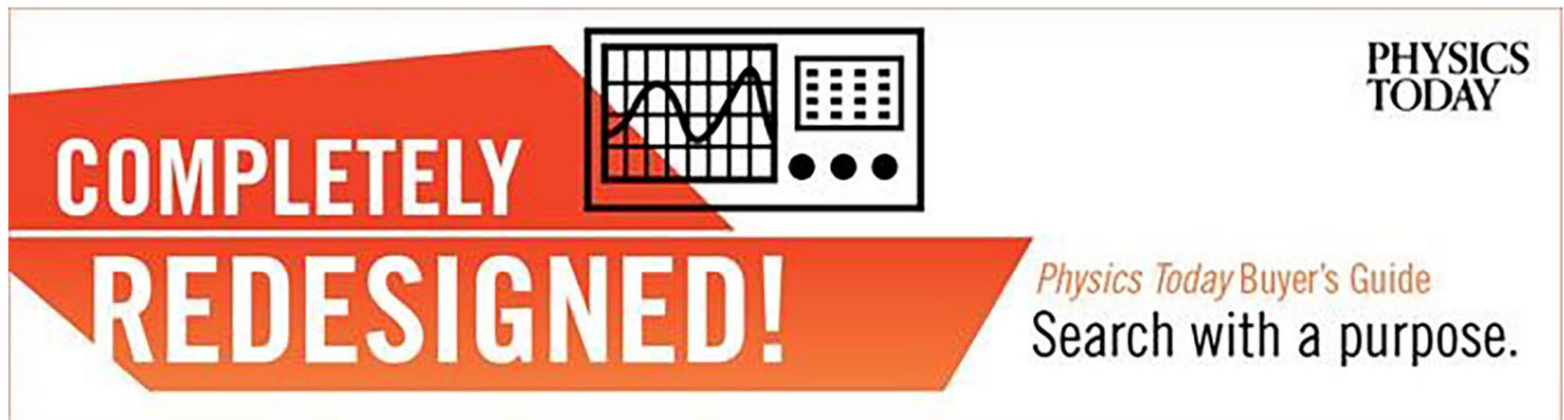




\title{
The role of hot electron refluxing in laser-generated $K$-alpha sources
}

\author{
P. Neumayer, ${ }^{1,2,3, a)}$ B. Aurand, ${ }^{1,4}$ M. Basko, ${ }^{5,3}$ B. Ecker, ${ }^{6,7,4}$ P. Gibbon, ${ }^{1,8}$ \\ D. C. Hochhaus, ${ }^{1,6,3}$ A. Karmakar, ${ }^{1,8}$ E. Kazakov, ${ }^{9}$ T. Kühl, ${ }^{6,4}$ C. Labaune, ${ }^{10}$ O. Rosmej, ${ }^{6}$ \\ An. Tauschwitz, ${ }^{1,3}$ B. Zielbauer, ${ }^{6,7}$ and D. Zimmer ${ }^{6,4,11}$ \\ ${ }^{1}$ ExtreMe Matter Institute EMMI, GSI Helmholtzzentrum für Schwerionenforschung, Planckstraße 1, \\ 64291 Darmstadt, Germany \\ ${ }^{2}$ FIAS Frankfurt Institute for Advanced Studies, 60438 Frankfurt am Main, Germany \\ ${ }^{3}$ Goethe Universität Frankfurt am Main, 60325 Frankfurt am Main, Germany \\ ${ }_{5}^{4}$ Johannes Gutenberg Universität Mainz, 55099 Mainz, Germany \\ ${ }^{5}$ Institute for Theoretical and Experimental Physics, 117218 Moscow, Russia \\ ${ }^{6}$ Helmholtzzentrum für Schwerionenforschung GSI, 64291 Darmstadt, Germany \\ ${ }^{7}$ Helmholtz Institut Jena, 07743 Jena, Germany \\ ${ }^{8}$ Institute for Advanced Simulation, Jülich Supercomputing Center, Forschungzentrum Jülich GmbH, \\ 52425 Jülich, Germany \\ ${ }^{9}$ RRC Kurchatov Institute, 123182 Moscow, Russia \\ ${ }^{10}$ LULI, Ecole Polytechnique, 91128 Palaiseau Cedex, France \\ ${ }^{11}$ Université Paris Sud 11, 91405 Orsay Cedex, France
}

(Received 3 May 2010; accepted 13 August 2010; published online 14 October 2010)

\begin{abstract}
A study of the contribution of refluxing electrons in the production of $K$-alpha radiation from high-intensity laser irradiated thin targets has been performed. Thin copper foils both freestanding, and backed by a thick substrate were irradiated with laser pulses of energies around $100 \mathrm{~J}$ at intensities ranging from below $10^{17}$ to above $10^{19} \mathrm{~W} / \mathrm{cm}^{2}$. At high laser intensities we find a strong reduction in the $K$-alpha yield from targets backed by the substrate. The observed yield reduction is in good agreement with a simple model using hot electron spectra from particle-in-cell simulations or directly inferred from the measured bremsstrahlung emission and can therefore be interpreted as due to the suppression of hot electron refluxing. The study shows that refluxing electrons play a dominant role in high-intensity laser driven $K$ - alpha generation and have to be taken into account in designing targets for laser driven high-flux K-alpha sources. ( 2010 American Institute of Physics. [doi:10.1063/1.3486520]
\end{abstract}

\section{INTRODUCTION}

High-intensity short-pulse laser-driven $K$-alpha sources offer a large potential for diagnostic applications in highenergy-density science experiments. The favorable scaling to higher energies allows efficient generation of x-rays well above $10 \mathrm{keV}$, suitable for probing matter at solid and supersolid density. ${ }^{1}$ The emission duration is closely linked to the hot electron relaxation and is of the order of picoseconds. As this time is short compared to the hydrodynamic evolution of the target, and the spatial distribution of the emission is weighted by the local density of the fluorescing material, using thin foils or wire targets source sizes as small as $10 \mu \mathrm{m}$ can be realized, enabling high-resolution point projection radiography with picosecond time resolution. ${ }^{2-4}$ Another field of application where the spectral quality of the $K$-alpha line emission is of importance is the technique of spectrally resolved x-ray Thomson scattering (for a recent review, see Ref. 5). Employing $K$-alpha probe radiation both the high temporal resolution and the satellite-free red wing in the spectrum enabled to clearly resolve the emergence of the plasmon resonance in coalescing shock experiments. ${ }^{6}$ All these unique properties make $K$-alpha sources the backlighter of choice for high rho-r plasmas such as shock-compressed solid density targets or the compressed fusion fuel in inertial

\footnotetext{
${ }^{a)}$ Electronic mail: p.neumayer@gsi.de.
}

confinement fusion (ICF) experiments. To provide the large photon numbers required for these applications, current highenergy density producing laser facilities have been or will be upgraded to feature multikilojoule short-pulse drive lasers. ${ }^{7-9}$

Equally important as using powerful drivers in achieving large photon numbers per shot is the conversion efficiency (CE), i.e., the ratio of energy of the generated $K$-alpha radiation to the input laser energy. When a high-intensity short laser pulse interacts with matter a considerable fraction of the laser energy is converted to supra-thermal electrons with relativistic energies. Propagating through the target material the electrons produce $K$-shell vacancies, leading to the emission of characteristic line radiation by radiative decay. The stopping range of these energetic electrons largely exceeds the attenuation length of the material at its own $K$-alpha photon energy, which is typically a few tens of micrometers. A large fraction of the radiation is therefore produced deep inside the target and will be reabsorbed if massive targets are used. When employing targets with dimensions of order of the attenuation length a significant number of hot electrons will reach the boundaries and leave the target carrying a considerable amount of energy. Reich et al. ${ }^{10}$ have pointed out that electrons leaving the target would create a strong space charge causing further hot electrons to return to the target, where they would create further $K$-alpha emission and consequently lengthen the $K$-alpha emission duration. This 
hot electron refluxing, or recirculation, was suggested by Sentoku et al. ${ }^{11}$ to explain the acceleration of protons from the surface of thin foil targets to several tens of MeV energies by the strong sheath fields. With a simple capacitance model Myatt et al. ${ }^{12}$ have estimated the refluxing efficiency, the fraction of hot electrons trapped by refluxing in the target, to $>90 \%$. The laser energy converted into this hot electron fraction is consequently confined to the target dimensions and experiments on reduced mass targets have shown the potential to generate samples of high energy density matter with moderate laser energies by reducing the target size. $^{13-15}$

Utilizing thin foils with thicknesses comparable to the attenuation length as targets for $K$-alpha sources CEs of laser energy into $K$-alpha radiation of $10^{-5}-10^{-4}$ are realized over a wide range of target materials, largely independent on the laser intensity and the target foil thickness (e.g., Refs. 1 and 16) and the transverse foil size, ${ }^{13}$ indicating a considerable contribution of refluxing electrons to the $K$-alpha yield. Modeling the $K$-alpha yield assuming full refluxing Theobald $e t$ $a l .{ }^{17}$ have reproduced the experimentally found independence of the CE on laser intensity. The model also predicts a drastic drop in $\mathrm{CE}$ at high laser intensity without the assumption of refluxing. This strongly reduced CE when suppressing hot electron refluxing has, to the best of our knowledge, not been experimentally verified or quantified. While the independence on intensity of the CE is merely a consequence of the assumed full refluxing and constant laser to hot electron energy conversion, and independent on the underlying hot electron distribution, the drop in $\mathrm{CE}$ with intensity when refluxing is suppressed is sensitive to details in the hot electron distribution.

In this work we present results studying the role of hot electron refluxing on the $K$-alpha yield from high-energy short pulse laser irradiated copper targets. Comparing freestanding foil targets and foils attached to a thick substrate, we observe large differences in the $K$-alpha yield at high laser intensities. We attribute these differences to the suppression of refluxing in the foils with substrate, which enables us to quantitatively determine the strong contribution of refluxing electrons to the generated radiation. This is supported by modeling of the $K$-alpha generation, which yields good agreement with the observed scaling.

\section{EXPERIMENTAL SETUP}

The experiment was performed at the PHELIX laser facility ${ }^{18}$ at the GSI Helmholtzzentrum für Schwerionenforschung GmbH in Darmstadt, Germany. A schematics of the experimental setup is shown in Fig. 1. Laser pulses with energies of $100 \mathrm{~J}$ and a pulse duration down to $0.7 \mathrm{ps}$ were focused onto the target using a $90^{\circ}$ off-axis parabola. The focal intensity distribution was imaged with a $10 \times$ magnification onto an 8-bit charge-coupled- device (CCD) camera. At best focus we measured a focal spot size of 20 $\times 30 \mu \mathrm{m}$ full width at half maximum (the slight ellipticity of the focal spot is due to the elliptical beam profile of the laser pulse behind the grating pulse compressor). We varied the on-target intensity while keeping the laser pulse energy

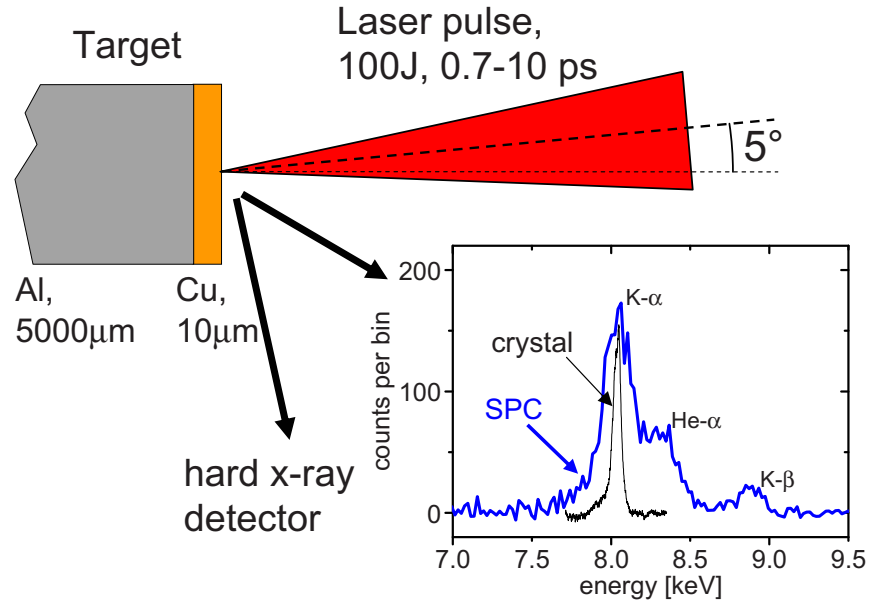

FIG. 1. (Color online) Schematics of the experimental setup and typical spectra obtained with the crystal spectrometer and the single-photon counting spectrometer.

constant by using pulse durations of 0.7 and $10 \mathrm{ps}$ and by defocusing the laser on the target. This allowed us to span a wide intensity range from $1.5 \times 10^{19}$ down to well below $10^{17} \mathrm{~W} / \mathrm{cm}^{2}$.

The laser was incident at an angle of $5^{\circ}$ onto the targets which were made of $10 \mu \mathrm{m}$ thick copper foil (transverse dimension $\approx 2 \mathrm{~mm}$ ). To investigate the effect of electron refluxing on the $K$-alpha yield, two types of targets were used. Besides freestanding foils, we used foils glued onto a several millimeter thick aluminum substrate. This served to absorb electrons leaving the rear side of the copper foil thus suppressing refluxing and charging up of the target by the escaping electrons is compensated by cold return currents. A lowviscosity carbon glue was used to minimize the distance between foil and substrate.

We have measured the copper $K$-shell emission spectra using a CCD camera in single-photon-counting mode. The setup consists of a cooled back-illuminated CCD (Princeton Instruments, $1024 \times 1024$ pixel) viewing the target at a distance of $3.3 \mathrm{~m}$ through a beryllium window. An evacuated vacuum tube up to the camera reduced absorption of low energy photons in air thus allowing detection of x-rays below $10 \mathrm{keV}$. Copper filters with a total thickness of $68 \mu \mathrm{m}$ were selected to operate in the single photon regime, such that on average much less than 1 photon per pixel is detected. As the charge produced by a photon in a pixel is approximately proportional to the photon energy, a spectrum is obtained from the histogram of the CCD exposure. We employed a single-event algorithm that discards events spread over more than one CCD pixel. The single-event detection efficiency is the probability of a photon to be detected multiplied with the probability that the charge produced is collected in a single pixel only. We determined the single-hit efficiency of the CCD camera at a photon energy of $5.9 \mathrm{keV}$ using $\mathrm{Mn}-K$ alpha radiation from a radioactive source $(\mathrm{Fe}-55)$. This value is then corrected by the difference in quantum efficiency as specified by the vendor to obtain the single-hit efficiency at the copper $K$-alpha photon energy of $8 \mathrm{keV}$. The activity of the radioactive calibration source is directly measured using 


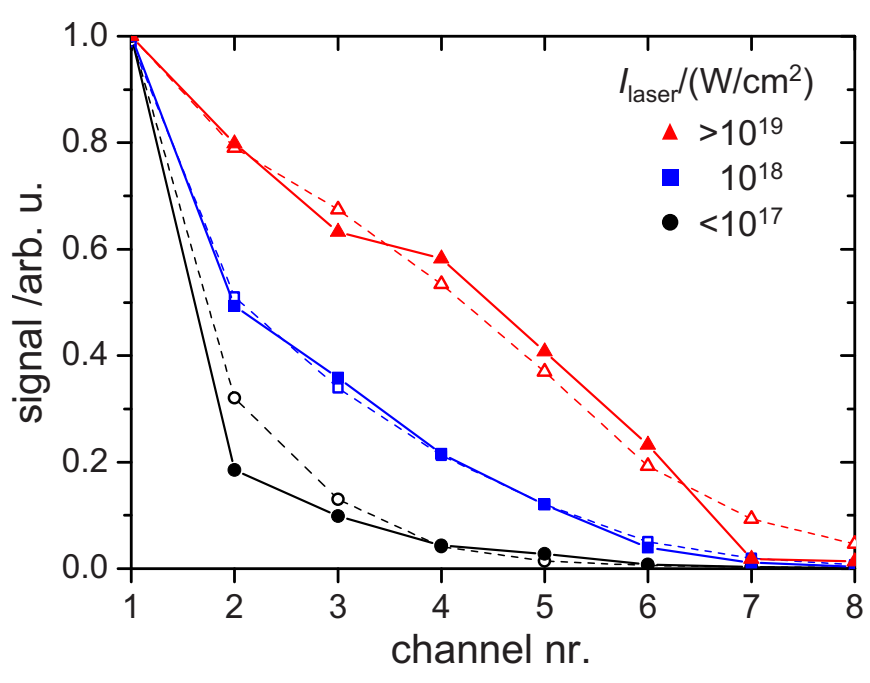

FIG. 2. (Color online) Signals measured in the eight channels of the HXRD (solid symbols) and best fit (open symbols) for intensities $<10^{17}, 10^{18}$, and $1.5 \times 10^{19} \mathrm{~W} / \mathrm{cm}^{2}$. The thickness of the lead filter increases with increasing channel number.

a PIN diode detector (XR-100T-CdTe, Amptek). We determined a single-event efficiency of $4 \%$ for $8 \mathrm{keV}$ photons providing an absolute calibration for the measurement of the $K$-alpha yield. A typical spectrum obtained from a $100 \mathrm{~J}$ shot is shown in Fig. 1. Besides strong $K$-alpha emission the spectra show $K$-beta and He-alpha radiation. The single-hit CCD spectrometer gives a broad spectral overview of the $K$-shell emission. In addition, the copper $K$-alpha emission was measured by a highly oriented pyrolytic graphite crystal spectrometer. A curved crystal was used in van Hamos geometry and the spectra were recorded on imaging plate detectors (Fujifilm). The spectrometer viewed the target front (laser) side at an angle of approximately $45^{\circ}$. The absolute efficiency of this second spectrometer was determined by crosscalibration to the single-hit CCD spectrometer.

As the energetic electrons produced in the intense lasermatter interaction propagate through the target material, collisions with target atoms lead to emission of bremsstrahlung radiation. The spectrum of the emitted bremsstrahlung can be used to extract information on the underlying hot electron distribution. We have measured the spectral distribution of this hard $\mathrm{x}$-ray continuum by the response on an imaging plate detector (Fujifilm, type BAS-MS) in eight separate channels. Each channel is filtered with a lead foil of different thickness ranging from $12 \mu \mathrm{m}$ to $1 \mathrm{~mm}$, constituting highpass filters with the filter edge at increasingly higher energies up to $300 \mathrm{keV}$. An additional $1 \mathrm{~mm}$ thick Teflon sheet serves to reduce background from high-energy electrons leaving the target. A massive brass collimator of $100 \mathrm{~mm}$ length was used to collimate the detector view to the target. The detector was set up at a viewing angle of $90^{\circ}$ to the foil normal.

Figure 2 shows the signal measured with this hard x-ray detector (HXRD) in each of the eight channels for shots at intensities $<10^{17}, 10^{18}$, and $1.5 \times 10^{19} \mathrm{~W} / \mathrm{cm}^{2}$, respectively. The filter thickness increases with increasing channel number. It can clearly be seen that with increasing intensity on target, the higher (i.e., more strongly filtered) channels obtain more and more signal. This means that the bremsstrahlung spectrum contains an increasing fraction of hard x-ray photons, indicating an increased number of high energy electrons in the target.

\section{RESULTS AND MODELING}

\section{A. Hot electron spectra}

The spectral information on the bremsstrahlung emission can be used to infer the distribution of the hot electron fraction inside the target. In experiments at laser intensities up to $5 \times 10^{18} \mathrm{~W} / \mathrm{cm}^{2}$, Beg et al. ${ }^{19}$ used a combination of PIN diodes and scintillators to determine the bremsstrahlung spectrum in five spectral windows ranging up to $250 \mathrm{keV}$ and found that the emission spectrum could not be fitted by a single temperature. Multichannel bremsstrahlung dosimeters based on thermoluminescence detectors or imaging plate detectors have been employed in intense laser-matter interaction experiments to determine the hot electron spectrum and the absolute conversion of laser energy into hot electrons. The hot electron distribution was inferred by using parametrized one- and two-temperature test distributions to fit the measured channel responses. ${ }^{20,21}$ Here we have used a genetic algorithm to determine the electron distribution underlying the measured response in the detector channels as produced by the emitted bremsstrahlung spectrum. The electron spectrum is sampled by a set of 17 discrete electron energies $E_{j}$ ranging from $20 \mathrm{keV}$ to $10 \mathrm{MeV}$. We calculated the bremsstrahlung spectrum $\phi_{j}(E)$ produced by an electron of energy $E_{j}$ recirculating in the target foil by Monte Carlo simulation using the EGSNRC code system ${ }^{22}$ for coupled transport of electrons and photons. With the filter transmission $t_{i}(E)$ in channel $i$ and the spectral sensitivity $S(E)$ of the imaging plate detector, the response in channel $i$ of the detector, produced by an electron of energy $E_{j}$ in the target, is given by $R_{i j}=\int \phi_{j}(E) t_{i}(E) S(E) d E$. The spectral sensitivity $S(E)$ of the imaging plate to photons of energy $E$ is assumed to be proportional to the energy deposited in the image plate sensitive layer which we determined by Monte Carlo simulations. The total response in channel $i$ is then the sum over all electron energies, $r_{i}=\sum_{j} R_{i j} N_{j}$, where $N_{j}$ is the number of electrons in energy bin $j$ with energy $E_{j}$.

Given a measurement $r_{i \text {,meas }}$ we now search for the electron distribution $N_{j}$ that best reproduces the measured result, i.e., that minimizes the rms distance between the measurement values and the simulation, $\sqrt{\sum_{i}\left(r_{i}-r_{i, \text { meas }}\right)^{2}}$. We perform the minimization employing a genetic algorithm. The distance between measurement and the simulated response is used as fitness parameter. We use a population size of 100 individuals, genetically represented by the number of electrons in each energy bin $j$. The initial population is randomly generated. Selection is realized by weighting the probability for reproduction with the combined fitness of two parent solutions. Inheritance from parent to children solutions is realized by genetic crossover, and mutation is realized by randomly adding or multiplying individual electron bins. The algorithm quickly converges and after a few thousand generations solutions only slightly improve further. In Fig. 2 the fittest solution $r_{i \text {,fit }}$ is shown (open symbols) together with 


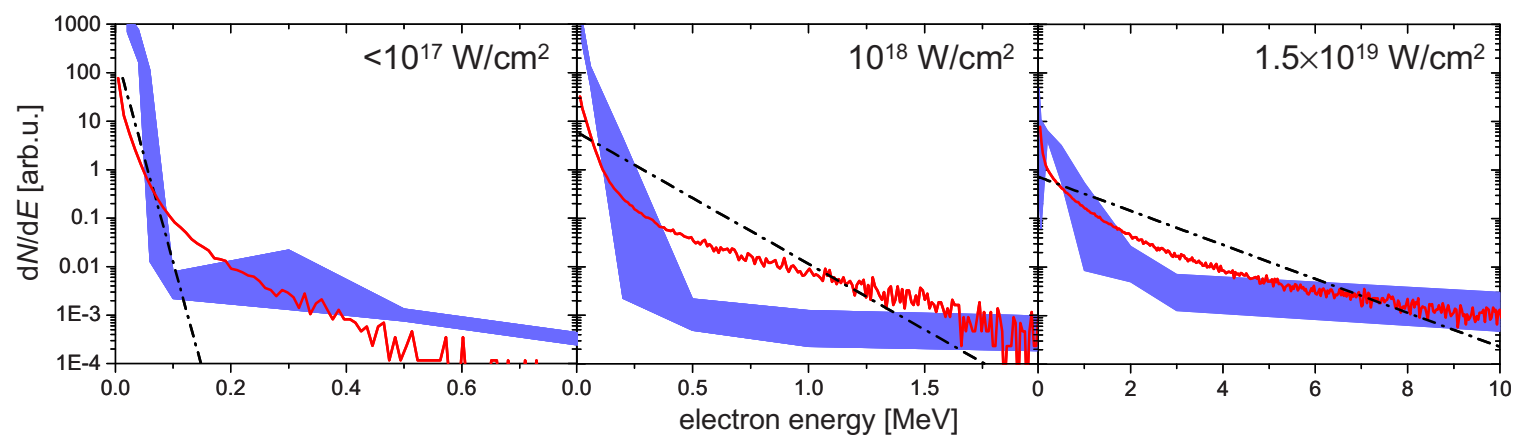

FIG. 3. (Color online) Hot electron distributions $d N / d E$ producing the best fit to the HXRD data (shaded areas), compared to 1-T distributions (dash-dotted line, black) and results from PIC calculations (solid line, red).

the measured channel responses $r_{i \text {, meas }}$ (solid symbols). A good fit to the data is obtained for all the intensities used in the experiment.

The electron distributions $N_{j} /\left(E_{j}-E_{j-1}\right)$ underlying these best fit results for shots at intensities of $<10^{17}, 10^{18}$, and $1.5 \times 10^{19} \mathrm{~W} / \mathrm{cm}^{2}$ are represented by the shaded areas in Fig. 3. The areas represent the boundaries of all solutions for different shots at a given intensity, as well as solutions from several individual optimization runs for each shot. Each solution has been normalized to a constant total energy. Note, that the boundaries are not solutions themselves. We clearly see a strong increase in the energetic electrons for increasing intensity. For the lowest intensity shots $\left(<10^{17} \mathrm{~W} / \mathrm{cm}^{2}\right)$ we basically find all the electrons, $99.6 \%$ of the total energy, at energies up to $50 \mathrm{keV}$. At intermediate intensities $\left(10^{18} \mathrm{~W} / \mathrm{cm}^{2}\right)$ this value drops to $80 \%$ and the remaining $20 \%$ are carried by electrons of up to $0.5 \mathrm{MeV}$ of energy. At the highest intensity $\left(>10^{19} \mathrm{~W} / \mathrm{cm}^{2}\right)$, electrons up to $1 \mathrm{MeV}$ energy carry $80 \%$ of the total energy, and still a significant fraction of $9 \%$ of energy is found in the highest energy bin at $10_{-2.5}^{+5} \mathrm{MeV}$. We compare the measured electron distributions with one-temperature distributions and with results from particle-in-cell (PIC) calculations. For the onetemperature (1-T) distributions $f(E) \propto \exp \left(-E / T_{\text {hot }}\right)$ (dashdotted line in Fig. 3), we have used the ponderomotive scaling by Wilks et al. ${ }^{23}$ to obtain the hot electron temperature $T_{\text {hot }}$ of 20,160 , and 1.2 MeV, respectively. The 1-T distributions clearly disagree with the electron spectra obtained from the HXRD measurement, which rather show a large colder electron fraction with a hot tail, consistent with observations of other authors (e.g., Refs. 19 and 21). The PIC calculations were performed in a density profile caused by the laser prepulse. We determined the preplasma conditions by twodimensional (2D) radiation-hydrodynamic modeling using the code RALEF-2D. ${ }^{24}$ Equation of state, thermal conductivity, and spectral opacities used were generated by the THERMOS code. $^{25}$ For the laser absorption, the analytical Kramers formula for the inverse bremsstrahlung was used. The laser pulse used for the calculations corresponds to the prepulse of the PHELIX laser system, which is mainly due to amplified spontaneous emission and was measured to consist of a 2 ns long pedestal with an intensity of $10^{-6}$ of the main pulse. The calculations predict a density scale length of $7 \mu \mathrm{m}$ at the critical density. PIC calculations for the foil target taking such preplasma were performed using the code BOPS. ${ }^{26}$ The code uses one spatial and three velocity dimensions and employs a Lorentz "boost" to emulate the oblique incidence. A total of $10^{6}$ electrons and ions were modeled on 10000 grid points. The laser was incident at $5^{\circ}$ and had a duration of 700 fs. The simulations predict a total conversion of laser energy into hot electrons of $2 \%, 7 \%$, and $14 \%$, for laser intensities of $10^{17}, 10^{18}$, and $1.5 \times 10^{19} \mathrm{~W} / \mathrm{cm}^{2}$, respectively. Hot electron spectra obtained from the PIC simulations are shown in Fig. 3, normalized to the same total energy as the measured (HXRD) and the 1-T distributions. In contrast to a 1-T distribution, the PIC simulated spectra show a colder and a hotter electron fraction. However, the cold electron fraction is still less pronounced than what we find in the measurement inferred from the HXRD. This could be explained by the fact that the PIC calculations are performed using only the peak intensity in the laser focus. The real focal intensity distribution however contains considerable fractions of laser energy over a whole range of intensities, up to the peak intensity. The expected electron distribution would thus rather consist of hot electrons produced at all these intensities, and would therefore contain a significant fraction of colder electrons produced at intensities lower than the peak intensity.

\section{B. K-alpha CE}

Figure 4 shows the measured $\mathrm{CE}$ into $K$-alpha for both free-standing foils and Al-backed foils for laser intensities ranging from below $10^{17}$ to $>10^{19} \mathrm{~W} / \mathrm{cm}^{2}$. We varied the intensity by variation of the pulse duration and focusing, while the laser pulse energy was kept constant to $100 \pm 20 \mathrm{~J}$. The measured $K$-alpha yields are normalized to the actual laser pulse energy to obtain the CEs, i.e., laser energy on target to energy into the $K$-alpha line. For the lowest intensity $\left(<10^{17} \mathrm{~W} / \mathrm{cm}^{2}\right)$ we measure CEs of $2 \times 10^{-5}-3 \times 10^{-5}$ for both foils with and without the aluminum substrate. When increasing the intensity to $10^{18} \mathrm{~W} / \mathrm{cm}^{2}$ the CE from the foils increases to $1 \times 10^{-4}-2 \times 10^{-4}$. The $\mathrm{CE}$ from the foil with aluminum substrate also increases, but stays a factor of 2-3 below that of the freestanding foil. This discrepancy becomes much more striking at intensities above $10^{19} \mathrm{~W} / \mathrm{cm}^{2}$. While the CE of the freestanding foils remains 


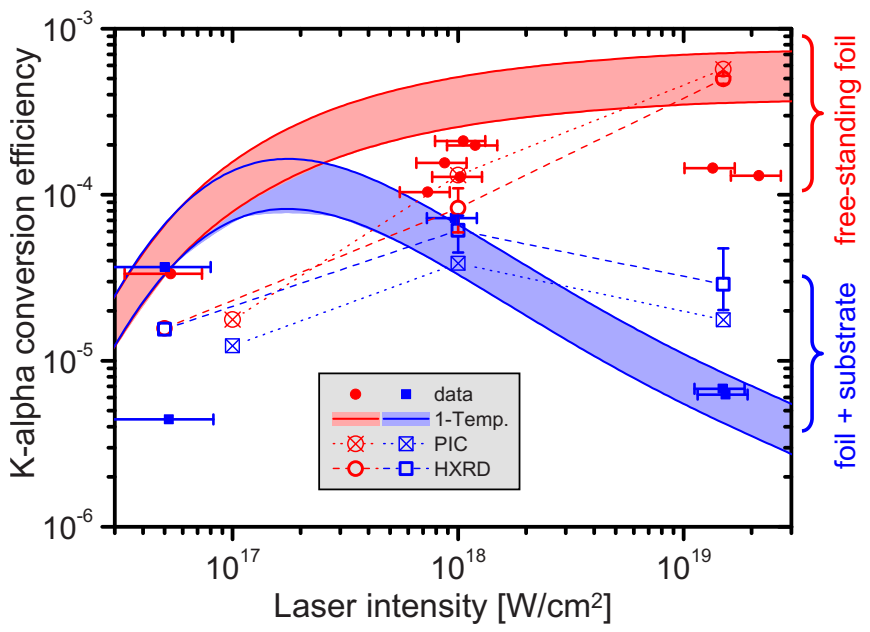

FIG. 4. (Color online) CE of laser energy into $K$-alpha radiation (into $4 \pi \mathrm{sr}$ ). Measurements (solid symbols) are compared with results from the model using various electron spectra: (solid line/shaded area) 1-T distribution with $\eta_{L \rightarrow e}=0.1-0.2$ and $T_{\text {hot }}$ obtained from the Wilks scaling, (crossed symbols) PIC simulations as described in the text, (open symbols) from the HXRD data. The two different targets are shown in red circles (refluxing target) and in blue squares (nonrefluxing target).

approximately constant around $10^{-4}$, the CE from the foils with aluminum substrate drops by more than an order of magnitude to $\approx 5 \times 10^{-6}$.

To show the crucial role of refluxing in explaining the measured $K$-alpha yields, we employ a model similar to the one discussed by Theobald et al. ${ }^{17}$ to the two types of targets used in this experiment. A hot electron fraction with a distribution function $f(E)$ and a total energy $E_{\mathrm{hot}}=\eta_{L \rightarrow e} E_{L}$ is sent into the copper foil starting at the front side. Here $E_{L}$ is the laser pulse energy and $\eta_{L \rightarrow e}$ the fraction of laser energy coupled into hot electrons. $K$-shell ionization is calculated using the ionization cross section $\sigma_{K}(E)$ obtained from the relativistic binary-encounter Bethe model ${ }^{27}$ which accounts for the relativistic rise at electron energies $>1 \mathrm{MeV}$. Energy loss of the electrons in the target is accounted for according to the stopping power $d E / d s$, obtained from the code ESTAR. ${ }^{28}$ For the refluxing target, the total number of $K$-alpha photons generated in the target is calculated by

$$
N_{K \alpha, \mathrm{refl}}=N_{e} n_{i} \omega_{K} \int_{0}^{\infty} d E_{0} f\left(E_{0}\right) \int_{0}^{E_{0}} d E \sigma_{K}(E)\left(\frac{d E}{d s}\right)^{-1},
$$

where $n_{i}$ is the target ion density and $\omega_{K} \approx 0.44$ is the $K$-alpha fluorescence yield for copper, i.e., the probability of a $K$-shell hole being filled by a radiative process, in competition with nonradiative processes. ${ }^{29}$ The total number of electrons $N_{e}$ follows from the normalization $E_{\text {hot }}$ $=N_{e} \int d E E f(E)$. Equation (1) assumes that electrons reaching either face of the target foil are reflected back into the target. Consequently the electrons remain inside the foil, producing $K$-shell ionization until their energy falls below the threshold for $K$-shell ionization. By contrast, for the nonrefluxing targets, we discard all electrons reaching the rear side of the foil after the first passage. The $K$-alpha yield is then calculated by

$$
N_{K \alpha, \text { nonrefl }}=N_{e} n_{i} \omega_{K} \int_{0}^{\infty} d E_{0} f\left(E_{0}\right) \int_{0}^{s_{0}} d s \sigma_{K}(E),
$$

where $d s=d E(d E / d s)^{-1}$. The path length of the electrons inside the target is given by the stopping length calculated in the continuous slowing down approximation, limited to the target thickness $d$, i.e., $s_{0}=\min \left[d, \int_{0}^{E_{0}} d s(d E / d s)\right]$.

To allow comparison to the measured CEs the yields predicted by the model are corrected for reabsorption of the $K$-alpha photons within the target foil on the way to the detector, using tabulated cold opacity and assuming a uniform hot electron density within the foil.

CEs calculated by this model are shown together with the experimental measurements in Fig. 4. These were obtained using three different hot electron energy distributions. Following the approach in Ref. 17 the solid lines are obtained using a 1-T Boltzmann-distribution, i.e., $f(E)$ $=1 / T_{\text {hot }} \exp \left(-E / T_{\text {hot }}\right)$ with the hot electron temperature $T_{\text {hot }}$ from the ponderomotive scaling. ${ }^{23}$ For the conversion of laser energy into hot electrons we used $\eta_{L \rightarrow e}=0.1-0.2$ (shaded area), which was inferred from the measurements presented in Ref. 14. This relatively simple model reproduces the $K$-alpha CEs to within about a factor of 4. Also shown in Fig. 4 are the CEs calculated by the model using the electron distribution function from the PIC calculations and those experimentally obtained from the HXRD. In the latter case the error bars are due to the range of electron distributions compatible with the HXRD data (shaded area in Fig. 3). The total number of electrons is obtained using the total energy in hot electrons $E_{\text {hot }}$ from the PIC calculations. For the intensity of $10^{18} \mathrm{~W} / \mathrm{cm}^{2}$ the model reproduces the measured CEs to within 50\% for both the refluxing and the nonrefluxing target types. At $1.5 \times 10^{19} \mathrm{~W} / \mathrm{cm}^{2}$ the model systematically overestimates the CEs. This might be attributed to additional energy loss mechanisms for the hot electron fraction at these intensities, that are not accounted for in the model, for example resistive return current effects or acceleration of ions from the target surface and consequently an adiabatic expansion of the hot electrons. ${ }^{15}$

Within our model, the ratio of $K$-alpha yields between the refluxing and nonrefluxing targets depends, for a given target material and thickness, only on the hot electron distribution function, more precisely on the ratio of low-energy electrons with a stopping range of order of the foil thickness, to energetic electrons with ranges that significantly exceed the foil thickness. In Fig. 5 we plot the ratio of the $K$-alpha CEs between the refluxing and the nonrefluxing targets. For the experimental results the values plotted are the ratios of the CEs averaged over all shots at a specific intensity, with the error being the standard deviation.

At the lowest intensities, the model predicts for all distribution functions used here no difference between refluxing and nonrefluxing targets, i.e., a ratio of 1 , in agreement with the experimental result. At the higher intensities of $10^{18}$ and $1.5 \times 10^{19} \mathrm{~W} / \mathrm{cm}^{2}$ the 1 - $\mathrm{T}$ distribution overestimates by about a factor 4 the drop in CE when refluxing is suppressed. On the other hand, the CE calculated from the distribution functions inferred from the HXRD data and from the PIC calculations are in good agreement with the experimentally 


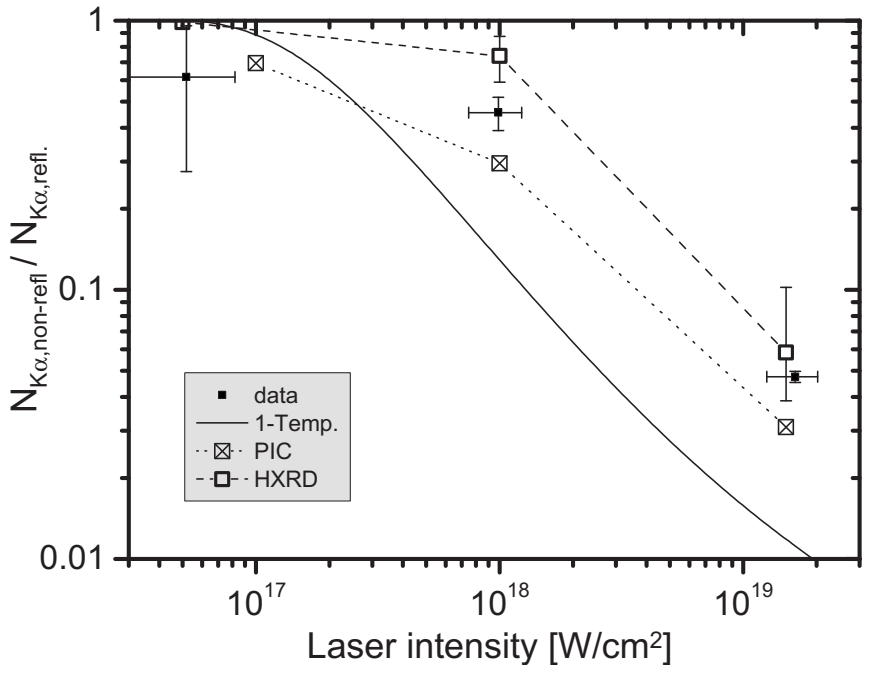

FIG. 5. Ratio of $K$-alpha CEs of the nonrefluxing to the refluxing target. For increasing intensity the CE drops by up to $20 \times$. The model prediction using a 1-T distribution (solid line) strongly overestimates the drop, while results using electron distributions from both PIC calculations (crossed symbols) and inferred from the HXRD data (open symbols) are in good agreement with the observed scaling.

found scaling. As pointed out above, the difference depends on the ratio of low energy electrons to the fraction of high energy electrons. At the high intensities of $>10^{19} \mathrm{~W} / \mathrm{cm}^{2}$ the total energy in electrons with ranges above $2 \times$ the foil thickness (i.e., electrons that will recirculate a significant number of times) is approximately $95 \%$ for the distributions obtained from the HXRD data and from the PIC calculations, while for the 1-T distribution it is $>99.5 \%$. For the intermediate intensity of $10^{18} \mathrm{~W} / \mathrm{cm}^{2}$ these electrons carry $10 \%$ (HXRD data) and 50\% (PIC) of the total energy, in contrast almost $90 \%$ in the $1-\mathrm{T}$ distribution.

\section{SUMMARY AND CONCLUSION}

In summary we have measured CEs of laser energy into $K$-alpha radiation from $10 \mu \mathrm{m}$ thick copper foils over a wide range of intensities. We have compared CEs from freestanding foils, where refluxing is expected to confine most of the laser-produced hot electrons inside the target foil, to foils backed by a thick substrate, which would suppress electron refluxing. We observed significant differences in the CE, which grow with increasing intensity. Modeling of the $K$-alpha generation inside the target using hot electron distributions from PIC calculations or inferred from the hard bremsstrahlung radiation is in good agreement with the observed scaling, supporting the interpretation that the differences in $\mathrm{CE}$ can be attributed to suppression of hot electron refluxing by the target substrate. A 1-T Boltzmanndistribution strongly overestimates this difference, suggesting that a larger fraction of low-energy electrons was present in the experiment. This is supported by the distribution functions measured in the experiment and found in PIC calculations.

At the highest intensities used in this experiment of 1.5 $\times 10^{19} \mathrm{~W} / \mathrm{cm}^{2}$, we measure a reduction of a factor of 20 when refluxing is suppressed, indicating that as much as about $95 \%$ of the $K$-alpha emission is generated by refluxing electrons. This fact has to be taken into account for efficient backlighter design. Suppression of refluxing, i.e., by buried layers or wires should be avoided. This is especially true when large photon numbers from small volumes are desired. The high intensities, inevitable especially when using high energy laser pulses, produce significant amounts of high energy electrons, which when not given the opportunity to recirculate, are lost for further $K$-alpha generation. On the other hand this might show a way to realize $K$-alpha sources with significantly shorter emission durations. For drive laser energies of several joule the $K$-alpha emission has been measured to be as long as $12-16 \mathrm{ps} .{ }^{30}$ The single-passage timeof-flight of an electron at the threshold energy for copper $K$-shell ionization through a $10 \mu \mathrm{m}$ thick foil, however, is below 200 fs. Suppression of hot electron refluxing could thus lead to significantly faster $K$-alpha sources, important e.g., when resolving ultrafast relaxation or phase transition phenomena. ${ }^{31,32}$

\section{ACKNOWLEDGMENTS}

We would like to thank the PHELIX laser and operations team for their support, as well as the GSI target laboratory for target preparation. This work was supported in part by Laserlab Europe and by the ExtreMe Matter Institute EMMI in the framework of the Helmholtz Alliance HA216/EMMI.

${ }^{1}$ H.-S. Park, D. M. Chambers, H.-K. Chung, R. J. Clarke, R. Eagleton, E. Giraldez, T. Goldsack, R. Heathcote, N. Izumi, M. H. Key, J. A. King, J. A. Koch, O. L. Landen, A. Nikroo, P. K. Patel, D. F. Price, B. A. Remington, H. F. Robey, R. A. Snavely, D. A. Steinman, R. B. Stephens, C. Stoeckl, M. Storm, M. Tabak, W. Theobald, R. P. J. Town, J. E. Wickersham, and B. B. Zhang, Phys. Plasmas 13, 056309 (2006).

${ }^{2}$ H.-S. Park, B. R. Maddox, E. Giraldez, S. P. Hatchett, L. T. Hudson, N. Izumi, M. H. Key, S. Le Pape, A. J. MacKinnon, A. G. MacPhee, P. K. Patel, T. W. Phillips, B. A. Remington, J. F. Seely, R. Tommasini, R. Town, J. Workman, and E. Brambrink, Phys. Plasmas 15, 072705 (2008). ${ }^{3}$ E. Brambrink, H. G. Wei, B. Barbrel, P. Audebert, A. Benuzzi-Mounaix, T. Boehly, T. Endo, C. D. Gregory, T. Kimura, R. Kodama, N. Ozaki, H.-S. Park, and M. Koenig, Phys. Rev. E 80, 056407 (2009).

${ }^{4}$ S. Le Pape, P. Neumayer, C. Fortmann, T. Döppner, P. Davis, A. L. Kritcher, O. Landen, and S. Glenzer, Phys. Plasmas 17, 056309 (2010).

${ }^{5}$ S. H. Glenzer and R. Redmer, Rev. Mod. Phys. 81, 1625 (2009).

${ }^{6}$ A. L. Kritcher, P. Neumayer, J. Castor, T. Döppner, R. W. Falcone, O. L. Landen, H.-J. Lee, R. W. Lee, E. C. Morse, A. Ng, S. Pollaine, D. Price, and S. H. Glenzer, Science 322, 69 (2008).

${ }^{7}$ C. P. J. Barty, M. Key, J. Britten, R. Beach, G. Beer, C. Brown, S. Bryan, J. Caird, T. Carlson, J. Crane, J. Dawson, A. C. Erlandson, D. Fittinghoff, M. Hermann, C. Hoaglan, A. Iyer, L. Jones II, I. Jovanovic, A. Komashko, O. Landen, Z. Liao, W. Molander, S. Mitchell, E. Moses, N. Nielsen, H.-H. Nguyen, J. Nissen, S. Payne, D. Pennington, L. Risinger, M. Rushford, K. Skulina, M. Spaeth, B. Stuart, G. Tietbohl, and B. Wattellier, Nucl. Fusion 44, S266 (2004).

${ }^{8}$ J. Schwarz, P. Rambo, M. Geissel, A. Edens, I. Smith, E. Brambrink, M. Kimmel, and B. Atherton, J. Phys.: Conf. Ser. 112, 032020 (2008).

${ }^{9}$ D. N. Maywar, J. H. Kelly, L. J. Waxer, S. F. B. Morse, I. A. Begishev, J. Bromage, C. Dorrer, J. L. Edwards, L. Folnsbee, M. J. Guardalben, S. D. Jacobs, R. Jungquist, T. J. Kessler, R. W. Kidder, B. E. Kruschwitz, S. J. Loucks, J. R. Marciante, R. L. McCrory, D. D. Meyerhofer, A. V. Okishev, J. B. Oliver, G. Pien, J. Qiao, J. Puth, A. L. Rigatti, A. W. Schmid, M. J. Shoup III, C. Stoeckl, K. A. Thorp, and J. D. Zuegel, J. Phys.: Conf. Ser. 112, 032007 (2008).

${ }^{10}$ Ch. Reich, P. Gibbon, I. Uschmann, and E. Förster, Phys. Rev. Lett. 84, 4846 (2000).

${ }^{11}$ Y. Sentoku, T. E. Cowan, A. Kemp, and H. Ruhl, Phys. Plasmas 10, 2009 (2003). 
${ }^{12}$ J. Myatt, W. Theobald, J. A. Delettrez, C. Stoeckl, M. Storm, T. C. Sangster, A. V. Maximov, and R. W. Short, Phys. Plasmas 14, 056301 (2007).

${ }^{13}$ S. D. Baton, M. Koenig, P. Guillou, B. Loupias A. Benuzzi- Mounaix, J. Fuchs, C. Rousseaux, L. Gremillet, D. Batani, A. Morace, M. Nakatsutsumi, R. Kodama, and Y. Aglitskiy, High Energy Density Phys. 3, 358 (2007).

${ }^{14}$ P. M. Nilson, W. Theobald, J. Myatt, C. Stoeckl, M. Storm, O. V. Gotchev, J. D. Zuegel, R. Betti, D. D. Meyerhofer, and T. C. Sangster, Phys. Plasmas 15, 056308 (2008).

${ }^{15}$ P. Neumayer, H. J. Lee, D. Offerman, E. Shipton, A. Kemp, A. L. Kritcher, T. Döppner, C. A. Back, and S. H. Glenzer, High Energy Density Phys. 5, 244 (2009).

${ }^{16}$ A. L. Kritcher, P. Neumayer, M. K. Urry, H. Robey, C. Niemann, O. L. Landen, E. Morse, and S. H. Glenzer, High Energy Density Phys. 3, 156 (2007).

${ }^{17}$ W. Theobald, K. Akli, R. Clarke, J. A. Delettrez, R. R. Freeman, S. Glenzer, J. Green, G. Gregori, R. Heathcote, N. Izumi, J. A. King, J. A. Koch, J. Kuba, K. Lancaster, A. J. MacKinnon, M. Key, C. Mileham, J. Myatt, D. Neely, P. A. Norreys, H.-S. Park, J. Pasley, P. Patel, S. P. Regan, H. Sawada, R. Shepherd, R. Snavely, R. B. Stephens, C. Stoeckl, M. Storm, B. Zhang, and T. C. Sangster, Phys. Plasmas 13, 043102 (2006).

${ }^{18}$ V. Bagnoud, B. Aurand, A. Blazevic, S. Borneis, C. Bruske, B. Ecker, U. Eisenbarth, J. Fils, A. Frank, E. Gaul, S. Goette, C. Haefner, T. Hahn, K. Harres, H.-M. Heuck, D. Hochhaus, D. H. H. Hoffmann, D. Javorková, H.-J. Kluge, T. Kuehl, S. Kunzer, M. Kreutz, T. Merz-Mantwill, P. Neumayer, E. Onkels, D. Reemts, O. Rosmej, M. Roth, T. Stoehlker, A. Tauschwitz, B. Zielbauer, D. Zimmer, and K. Witte, Appl. Phys. B: Lasers Opt. 100, 137 (2009).

${ }^{19}$ F. N. Beg, A. R. Bell, A. E. Dangor, C. N. Danson, A. P. Fews, M. E. Glinsky, B. A. Hammel, P. Lee, P. A. Norreys, and M. Tatarakis, Phys. Plasmas 4, 447 (1997).

${ }^{20}$ H. Schwoerer, P. Gibbon, S. Düsterer, R. Behrens, C. Ziener, C. Reich, and R. Sauerbrey, Phys. Rev. Lett. 86, 2317 (2001).

${ }^{21}$ C. D. Chen, P. K. Patel, D. S. Hey, A. J. Mackinnon, M. H. Key, K. U. Akli, T. Bartal, F. N. Beg, S. Chawla, H. Chen, R. R. Freeman, D. P. Higginson, A. Link, T. Y. Ma, A. G. MacPhee, R. B. Stephens, L. D. Van Woerkom, B. Westover, and M. Porkolab, Phys. Plasmas 16, 082705 (2009).
${ }^{22}$ I. Kawrakow and D. W. O. Rogers, "The EGSNRC Code System: Monte Carlo Simulation of Electron and Photon Transport," NRCC Report PIRS701, 2006.

${ }^{23}$ S. C. Wilks, W. L. Kruer, M. Tabak, and A. B. Langdon, Phys. Rev. Lett. 69, 1383 (1992).

${ }^{24}$ M. M. Basko, J. Maruhn, and An. Tauschwitz, "Development of a 2D radiation-hydrodynamics code RALEF for laser plasma simulations," GSI Scientific Report 2009, GSI Report 2010-1, 2009.

${ }^{25}$ A. F. Nikiforov, V. G. Novikov, and V. B. Uvarov, Quantum-Statistical Models of Hot Dense Matter: Methods for Computation Opacity and Equation of State (Birkhäuser Verlag, Basel-Boston-Berlin, 2005).

${ }^{26}$ P. Gibbon, A. Andreev, E. Lefebvre, G. Bonnaud, H. Ruhl, J. Delettrez, and A. R. Bell, Phys. Plasmas 6, 947 (1999).

${ }^{27}$ J. P. Santos, F. Parente, and Y.-K. Kim, J. Phys. B 36, 4211 (2003).

${ }^{28}$ M. J. Berger, J. S. Coursey, M. A. Zucker, and J. Chang, ESTAR, PSTAR, and ASTAR: Computer Programs for Calculating Stopping-Power and Range Tables for Electrons, Protons, and Helium Ions (version 1.2.3) (National Institute of Standards and Technology, Gaithersburg, 2005).

${ }^{29}$ M. O. Krause, J. Phys. Chem. Ref. Data 8, 307 (1979).

${ }^{30}$ H. Chen, R. Shepherd, H. K. Chung, A. Kemp, S. B. Hansen, S. C. Wilks, Y. Ping, K. Widmann, K. B. Fournier, G. Dyer, A. Faenov, T. Pikuz, and P. Beiersdorfer, Phys. Rev. E 76, 056402 (2007).

${ }^{31}$ C. W. Siders, A. Cavalleri, K. Sokolowski-Tinten Cs. Tth, T. Guo, M. Kammler, M. Horn von Hoegen, K. R. Wilson, D. von der Linde, and C. P. J. Barty, Science 286, 1340 (1999).

${ }^{32}$ A. M. Lindenberg, J. Larsson, K. Sokolowski-Tinten, K. J. Gaffney, C. Blome, O. Synnergren, J. Sheppard, C. Caleman, A. G. MacPhee, D. Weinstein, D. P. Lowney, T. K. Allison, T. Matthews, R. W. Falcone, A. L. Cavalieri, D. M. Fritz, S. H. Lee, P. H. Bucksbaum, D. A. Reis, J. Rudati, P. H. Fuoss, C. C. Kao, D. P. Siddons, R. Pahl, J. Als-Nielsen, S. Duesterer, R. Ischebeck, H. Schlarb, H. Schulte-Schrepping, Th. Tschentscher, J. Schneider, D. von der Linde, O. Hignette, F. Sette, H. N. Chapman, R. W. Lee, T. N. Hansen, S. Techert, J. S. Wark, M. Bergh, G. Huldt, D. van der Spoel, N. Timneanu, J. Hajdu, R. A. Akre, E. Bong, P. Krejcik, J. Arthur, S. Brennan, K. Luening, and J. B. Hastings, Science 308, 392 (2005). 